\title{
Intrauterine Gel Dosage Form
}

National Cancer Institute

\section{Source}

National Cancer Institute. Intrauterine Gel Dosage Form. NCI Thesaurus. Code C156477.

Semi-solid preparation consisting of a gel intended for intrauterine use. 